\title{
Anthroponymie shakespearienne
}

Jean Dubu

\section{(2) OpenEdition \\ Journals}

Édition électronique

URL : http://journals.openedition.org/shakespeare/1271

DOI : 10.4000/shakespeare.1271

ISSN : 2271-6424

Éditeur

Société Française Shakespeare

Édition imprimée

Date de publication : 1 novembre 1994

Pagination : 57-66

Référence électronique

Jean Dubu, «Anthroponymie shakespearienne », Actes des congrès de la Société française Shakespeare [En ligne], 12 | 1994, mis en ligne le 01 janvier 2007, consulté le 05 mai 2019. URL : http:// journals.openedition.org/shakespeare/1271 ; DOI : 10.4000/shakespeare.1271

(c) SFS 
COSMOPOLITISME ET INSULARITÉ 


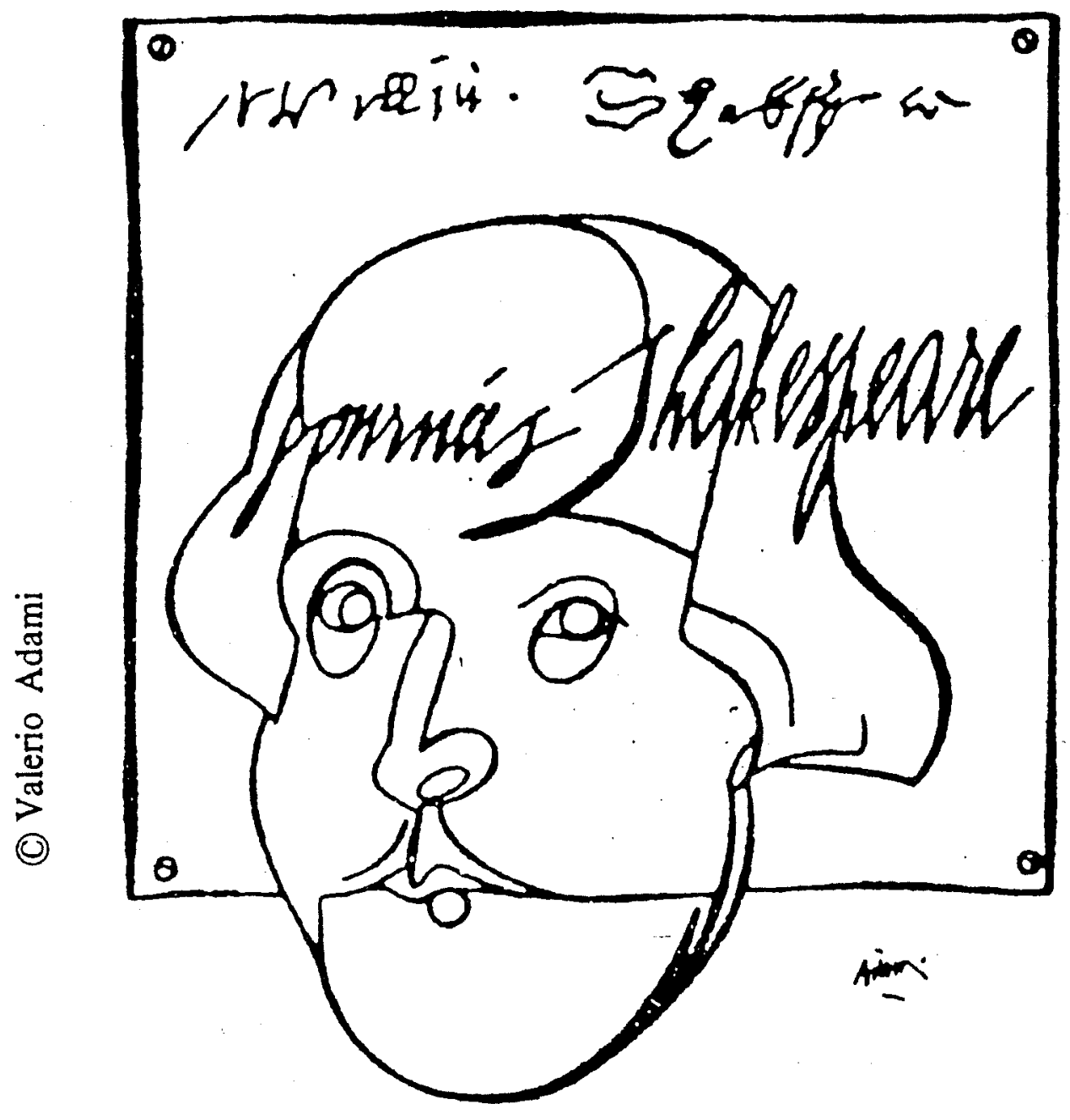

Affiche de Valerio Adami, spécialement conçue pour les journées Shakespeare 1979 


\section{SHAKESPEARE}

COSMOPOLITISME ET INSULARITÉ

Société Française Shakespeare

Actes du Congrès 1993

sous la direction

de

M. T. JONES-DAVIES

Ouvrage publié avec le soutien de

BARCLAYS

BARCLAYS BANK S.A

PARIS

LES BELLES LETTRES

1994 
Tous droits de traduction, de reproduction et d'adaptation réservés pour tous les pays.

(C) 1994 Société d'édition Les Belles Lettres, 95 bd Raspail 75006 Paris

ISBN 2.251.69122-7 


\section{Anthroponymie shakespearienne}

L'insertion de personnages nouveaux dans une intrigue ou une fable déjà connue constitue un usage courant chez les écrivains, a fortiori chez les dramaturges pressés par le temps et par le souci d'animer des épisodes qu'ils n'ont pas le loisir de raconter ; c'est aussi une manière de soutenir l'intérêt du public, de piquer sa curiosité ; d'une simple remarque, des figures neuves amènent parfois les héros à dévoiler leurs intentions, à faire le point de leurs espoirs ou de leurs désillusions. Tel est le propre des confidents de toute sorte, et plus généralement de ces personnages adventices que l'on nomme des utilités.

La désinvolture apparente avec laquelle Shakespeare les introduit et les nomme mérite de retenir notre attention, de même que le soin qu'il apporte à modifier par le truchement du patronyme l'identité et donc la personne, le caractère de tel de ses héros et héroïnes; une fois cette direction prise, il sait tirer parti des noms qu'il ne modifie pas, mais qu'il s'emploie à mieux mettre en valeur, en exploitant les richesses sémantiques qu'ils recêlent. L'attention aux mots est le propre des poètes. Nous nous proposons de présenter ici quelques remarques, nécessairement brèves, sur cette anthroponymie shakespearienne; du haut en bas de la société, des bas-fonds de Londres ou de Famagouste aux trônes d'Elseneur ou d'Egypte, elle permet à l'auteur de soutenir l'intérêt de son public, d'acclimater des thèmes venus de fort loin, d'enrichir la légende ou l'histoire nationale en les rapprochant de ses contemporains, et le patrimoine humain par des notations qui relèvent du simple bon sens.

Ce far presto l'entraîne souvent à désigner les personnages secondaires par leur fonction.

Ainsi, dans 1 Henry $I V$, nombre d'utilités (vintner, carriers ...) ne sont pas "nommées"; mais certaines éditions attestent que Bardolph et Peto, entre autres, ont changé d'appellation au cours des âges. La Nourrice de Juliette n'a pas été mieux traitée : elle n'a pas de nom propre ; alors que son valet répond au prénom bien anglais de Peter, les autres domestiques des Capulets ont nom Sampson, Gregory, Anthony et Potpan. L'auteur ne se met guère 
en frais de couleur locale. La tendance serait plutôt à rapprocher du public du pit, autochtone, les gens du commun pour les rendre plus directement accesssibles, en se fiant par contraste au caractère plus internationalement homogène des bourgeois et des grands de ce monde, afin de signifier la distance sociale; dans les deux cas, Shakespeare tend à faciliter le processus d'identification des uns et des autres avec leurs semblables, héros ou non, moyen si propre à susciter l'intérêt du public. Dans la suite des deux parties de Henry $I V$ et dans Henry $V$, le personnage récurrent de la tenancière de la taverne, accède au degré minimal de caractérisation, par le biais de son nom, Mistress Quickly, d'ailleurs aimablement nuancé à l'occasion quand on l'entend interpeller sous le vocable Dame Partlet the hen, dépourvu d'ambigüité 1 .

Dans le domaine des grands de ce monde, un rapprochement assez savoureux nous est procuré par un texte de Montaigne, bien que nous soyons certains que Shakespeare n'ait pu en avoir connaissance puisqu'il s'agit du "Journal de Voyage", qui ne devait être publié qu'un siècle et demi plus tard. Lors du passage de l'auteur des Essais à Vérone, celui-ci fait mention des Seigneurs de $l^{\prime} E s c a l e^{2}$, et cette dénomination correspond assez bien à "Escalus, prince of Verona", que nous trouvons dans le Dramatis personae de Romeo \& Juliet. De fait, à Vérone, on parlait surtout, on parle encore, de la famille Scaliger, mais depuis que les Della Scala (Jules-César le père, et Joseph-Juste le fils) érudits et controversistes, le second protestant, avaient adopté pour patronyme savant la version latine "Scaliger", non sans laisser entendre qu'ils étaient apparentés aux illustres seigneurs véronais, une atmosphère de dérision entourait ce mot latin, d'autant plus que l'antipathie déclarée de Joseph-Juste vis-à-vis des Anglais ${ }^{3}$ n'arrangeait rien OutreManche ; bref, dans l'intérêt de sa tragédie, Shakespeare évite de l'employer et préfere Escalus/ de l'Escale; dans ce respect verbal, et légitime de l'autorité constituée, il se retrouve en la compagnie de Montaigne. Si donc la vraisemblance est ici sauvegardée, nous ne devons pas oublier que le patronyme d'Escalus reparaît en 1604, dans Measure for Measure, dont l'action se déroule à Vienne, où il désigne "an ancient lord" selon le Dramatis personae, mais le personnage est nommé dans le texte, alors que le nom du prince de Vérone ne fait pas partie du texte de Romeo \& Juliet. La même année 1604 , le nom d'Escalus apparaît une dernière fois sous les murs de Florence, et désigne un comparse : le mot est entré au magasin des accessoires, il n'en ressortira pas. 
En revanche, dans Romeo \& Juliet, le nom du héros éponyme offre la possibilité d'une manière de caractérisation, en le prenant au pied de la lettre comme le terme qui désigne le pélerin retour de Rome, le "roumieux"; Shakespeare ne consacrera pas moins d'un sonnet, celui de la déclaration, à une précieuse variation sur le thème du pélerin qui transforme Romeo en émule du viator amoris cher aux troubadours. Dans The Merchant of Venice, si le comparse Tubal est suffisamment caractérisé par ce nom emprunté à l'Ancien Testament le plus reculé, l'auteur semble bien s'en remettre à un calembour pour désigner Shylock, celui qui ne parvient pas à tenir suffisamment enfermés ses deux trésors : son or et sa fille.

Si par ailleurs, pour ce qui était, primitivement, "Le More de Venise", l'auteur choisit d'une manière déconcertante des noms plutôt ibériques d'origine, à commencer par celui du héros éponyme, Othello, tel Iago et même Roderigo, bien que ce dernier soit décrit comme "gentilhomme vénitien", c'est sans doute qu'il convient de faire prévaloir l'atmosphère méditerranéenne; mais aussi dans cette oeuvre si profondément émouvante, dramatique, qui s'achève par le meurtre d'une innocente, ne convenait-il pas de rappeler quelque peu l'univers si largement applaudi en son temps de The Spanish Tragedy? L'évocation discrète d'un ailleurs déjà connu mène l'auteur à préférer purement et simplement un exotisme de culture, qui ne risque pas de désorienter le public, en ne le dépaysant que ce qu'il faut par le biais du nom des protagonistes.

Que dire alors de Brabantio, sénateur "vénitien", père de l'héroïne ? Ne s'agirait-il pas là du rappel d'une piste onomastique qui nous conduit vers la province de BRABANT, aux Pays-Bas ? Cette piste nous semble avoir sa place dans l'esprit (d'aucuns diraient sans doute dans l'inconscient ...) de notre auteur : nous aurons l'occasion de le voir à propos de la mère de Hamlet, la reine Gertrude.

Un second aspect de l'utilisation que Shakespeare sait faire de la valeur des noms de personne se présente alors à nous : nous en trouvons un exemple flagrant dans les noms de certains des héros de King Lear, à commencer par le roi lui-même. Les diverses sources, y compris les plus récentes, soit Edmund Spenser et l'auteur inconnu de King Leir orthographient le nom du Roi : Leir, ce que corrobore l'orthographe de la ville de Leicester, considérée comme la forteresse et la cité du souverain. Mais Shakespeare préfêre l'écrire comme vous savez, ce qui, en une espèce de backslang, 
peut se lire real et se comprendre soit comme le français réal, équivalent de royal,et confirme l'un des messages de la pièce, celui qu'entend si bien Cordelia lors de son retour : le Roi se doit d'être royal, et cela fait partie de sa prise sur le "réel" (real), inhérente au personnage politique que, sénile ou pas, il ne peut s'empêcher d'être. Toute forme d'abdication lui est interdite : la morale politique de cette tragédie rejoint celle de King Richard II dans son exigence personnelle à l'égard du souverain; d'autre part King Lear est écrit lorsque le roi Jacques vient d'accéder au trône d'Angleterre, dans des circonstances délicates, et le prince ne fait pas mystère du but qu'il se propose d'atteindre sur le plan intérieur: la fusion de ses divers royaumes en un seul état, qui serait le Royaume de Grande-Bretagne. Comme nous le remarquions voici dix ans devant cette Sociétét, les monnaies frappées la seconde année du règne de Jacques Ir portent l'inscription Britanniae, Franciae \& Hiberniae Rex qui signifie clairement les intentions du souverain en la matière. Serait-ce alors un hasard ou une inadvertance de la part de Shakespeare, si les deux gendres du roi deviennent l'un duc de Cornouailles, et l'autre duc d'Albany, c'est-à--dire les titulaires, le premier de l'apanage traditionnel de l'héritier de la couronne anglaise et le second de celui de la couronne écossaise, alors que Cordelia épouse le roi de France, dont le royaume est toujours revendiqué à l'époque par les rois d'Angleterre, et ce depuis 1328 ? Autant dire que le dramaturge dont la troupe a été pensionnée par le roi Jacques dès l'arrivée de celui-ci à Londres, rebaptise ces deux personnages dans un sens qui vient à point nommé servir la politique de son protecteur.

Depuis La Princesse de Clèves on sait quel devint très rapidement l'ascendant de la Cour des Valois en Europe sous François $\mathrm{I}^{\mathrm{er}}$ : l'étiquette en fut codifiée par les soins de son petit-fils Henri III. Shakespeare ne l'ignore pas, même s'il y trouve matière à ironiser. Ainsi dans The Merchant of Venice, l'interrogation malicieuse de Nerissa à Portia :

How say you by the French lord, Monsieur Le Bon?

qui situe parfaitement le personnage. Puisque la pastorale suppose une vie de Cour, on n'est pas surpris de la récurrence du terme Monsieur dans As You Like it : 11 occurrences, avec une progression instructive; à la scène 5 de l'acte II, Amiens refuse de poursuivre sa chanson :

It will make you melancholy, Monsieur Jaques. 
A l'acte suivant, son frère Orlando tirera un sobriquet de nature de cette épithète, et Jaques devient :

I am glad of your departure; adieu, good Monsieur Melancholy.

Au quatrième acte, le départ du même Jaques est salué par Rosalind d'un non moins ironique :

Farevell, Monsieur Traveller.

et l'on conserve en mémoire le Monsieur Paroiles, de All Is Well That Ends Well, - Monsieur Parolles dont la réputation est précisément d'être a notorious liar, c'est-à-dire de ne pas tenir parole. D'autre part, il ne s'agit nullement d'une erreur typographique lorsque nous lisons ce qui, de toute évidence, ne peut être que le même terme, orthographié mounsieur, au début de la première scène de l'acte IV de A Midsummer Night's Dream. Trait de réalisme verbal, ce défaut de prononciation vient renforcer le ridicule des artisans, qui singent la Cour dans ses divertissements et jusque dans son langage. La titulature ainsi déformée, jointe aux termes très familiers de Cobweb, Mustardseed \& al. produit un effet burlesque, alors que les divers surnoms appliqués à Jaques (Melancholy, Traveller) le caractérisent parfaitement. Le même procédé se trouve dans I Henry $I V$, l'ironie en plus, lorsque Poins s'adresse à Falstaff en ces termes : Sugar?

What says Monsieur Remorse? What says Sir John Sack \&

Dans le même ordre d'idées, ne trouvons-nous pas dans Pericles un Monsieur Verollus sur lequel il semble inutile d'épiloguer : l'évidence du terme s'impose.

Si le nom de Claudio désigne un jeune seigneur de Florence dans Much Ado About Nothing, et un jeune gentilhomme dans Measure For Measure, le Feng ou Fengon de Saxo Grammaticus laisse place à Claudius dans le Dramatis personae de Hamlet, Prince of Denmark, et ce nom semble venir tout droit de The Murder of Gonzago ; mais il est remarquable que Shakespeare, ne le désigne dans le texte que par sa fonction, The King. En revanche, les noms des autres personnages de la tragédie peuvent être classés en trois catégories et l'on peut essayer de les définir. Les mots d'origine scandinave conservés, Hamlet, Fortinbras, Valtemand, Osric, même si, tel Hamlet ils ont été modifiés pour les angliciser légèrement. Rozenkrantz, Guildenstern, Cornelius sont associés à la fois à Wittenberg, où un registre de l'Université at- 
teste encore la présence des deux premiers au nombre des étudiants. Malgré sa consonance italienne, il faut y joindre le nom d'Horatio : l'origine latine du mot est évidente. Ce ne sont pas des noms de princes, mais de lettrés, voire de diplomates, de jeunes courtisans d'avenir, bref d'hommes qui ont reçu une éducation, et une instruction susceptibles de les faire accéder à certains emplois. Cette forme d'autorité investit Marcellus au plus bas niveau, et Polonius au plus élevé, ainsi que, d'une certaine manière Laertes. Les personnages de rang inférieur ont des noms italiens. Et les femmes? Comme vous le savez, leur nombre est réduit à deux : le nom d'Ophelia est-il une allusion solaire à la fair temptress de la légende ? On serait tenté de le penser. Et Gertrude ?

GERTRUDE ! Certes c'est la forme francophone de l'antique GERUTH. Ou GERUTHA danois. C'en est aussi l'antithèse, en ce que la partie francophone des Pays-Bas a donné en Wallonie, à Nivelles, une sainte Gertrude, aux rats, et ici je, ne peux mieux faire que de citer un livre tout récent, achevé d'imprimer en juin 1992, dû à M. Jacques Berchtold notre collègue à Princeton :

Sainte Gertrude, qui vécut à Nivelles de 631 à 659 , et dont la Vita a fait l'objet de récits en latin dès le VII ${ }^{e}$ siècle, devient soudainement, dès le début du second tiers du $X^{e}$ s., la patronne privilégiée des rats et des souris, en Alsace, en Catalogne, en Brabant, dans tous les Pays-Bas, en Rhénanie et en Autriche. Cette attribution n'a de fondement dans aucun épisode de la biographie de la sainte; son origine est généralement recherchée dans le folklore populaire ${ }^{5}$.

En fait, et pour schématiser, des œuvres de saint Augustin et de la tradition, il ressort un concept de la femina muscipula, véritable "ratière", dont l'homme devient aisément la proie. On comprend, je l'espère, sans qu'il soit besoin d'y insister la nature du piège et le symbole que représente le muscidé dans cette affaire. Or, si l'abbesse Gertrude de Nivelles, morte en 659 , est devenue "sainte" Gertrude, et la protectrice des souris et des rats, c'est à cause de sa virginité préservée. Un vitrail du musée de Strasbourg et de nombreuses gravures reproduites par notre collègue dans son livre, montrent la sainte abbesse, soit lisant, soit filant à son aise, entourée des parasites domestiques apprivoisés qui ne cherchent à lui faire aucun mal, et à qui elle n'en fait pas. Il va de soi qu'en regard, la reine Gertrude, instigatrice de l'assassinat de son mari par adultère, et, selon Belleforest, maîtresse de Fengon/Claudius, avant la mort de Amleth le père, apparaît typique de la femina muscipula, et donc l'antithèse de la sainte de Nivelles, son homonyme. 
On n'en comprend que mieux la réaction de Hamlet lorsqu'il voit remuer la tapisserie dans le boudoir de sa mère : "A rat! A rat !", comme aussi bien le titre de la pantomime The Mouse-trap, car dans le système d'explication adopté rats et souris, c'est tout un ; mais, laissons derechef la parole à J. Berchtold :

Durant la présentation des deux portraits royaux, la présence du cadavre de Polonius (i.e. la présence du rat) continue de faire signe sur la scène. Le cadavre de Polonius (i.e. du rat) reste étendu sur la scène durant tout le dialogue entre Hamlet et Gertrude avant d'être enlevé et apparaît comme l'indice d'un "memento mori". Hamlet et Gertrude discourent sur la fidélité et sur l'amour durant deux cents vers, en paraissant avoir oublié la présence du cadavre qui gît à leurs pieds. Le cadavre de Polonius (i.e. le rat mort), comme le crâne au bas du tableau des Ambassadeurs de Holbein, est l'indice d'un "memento mori" présent dans la chambre à coucher stigmatisée comme espace de luxure. ${ }^{6}$

Et M. Berchtold de conclure quelques lignes plus loin :

A la fin de son sermon, Hamlet peut enfin débarrasser la scène du cadavre (i.e. du rat) et convier Gertrude à la continence et à la repentance : «Good night. But go not to my uncle's bed» (III, 4,161).

On a pu mesurer avec quelle pertinence notre collègue s'applique à nous montrer comment Shakespeare transforme la reine de Danemark en une Gertrude de sens contraire, par rapport à l'abbesse de Nivelles, et justifie par là-même le choix de la version francophone du nom de l'héroïne.

Conclusion logique de ce premier choix, Hamlet devient un émule de l'homme aux rats amplement connu au Moyen-Age et à la Renaissance, et sur lequel Freud a longuement glosé. Ce terrain n'est pas celui sur lequel je désire m'aventurer. Toutefois, notre collègue attire aussi notre attention dans son livre sur le chapitre III des Histoires prodigieuses de Boaistuau où est rapportée l'histoire du Roi Popiel de Pologne, homme "méchant" qui finit dévoré par les rats ${ }^{7}$. Or cette histoire de Boaistuau est reprise par Belleforest que Shakespeare connaît et utilise. C'est ainsi que s'expliquerait, notamment, que le dramaturge inscrive dans l'histoire du prince Amleth "une sorte de palimpseste faufilé de l'Histoire du Roi Popiel"8. (Je laisse à notre collègue la responsabilité du fauxsens "genettique" sur "palimpseste"). Mais de cette corrélation des deux histoires proviendrait la modification du nom (et cela nous ramène au cœur de notre propos) de Corambis/Polonius. L'un des 
points délicats de l'anthroponymie shakespearienne n'est-ce pas le changement du nom de Corambis imprimé dans le Premier Quarto en Polonius dans le Second? Si nous admettons l'hypothèse qu'adopte notre collègue, de par les liens de fonction de ce personnage auprès du roi, Corambis/Polonius devient un double de Claudius ; sa connivence intéressée à la promotion de sa fille au rôle de "fair temptress" ne peut que renforcer son partage de culpabilité avec le nouveau souverain, et donc sa vocation à une fin ignominieuse, du genre de celle que subit Popiel de Pologne. Le cri de Hamlet "A rat! A rat !" le rend coupable. On verra de plus, si l'on prend le loisir d'examiner en détail, - ce que je n'ai pas le temps de faire ici - l'argumentation de J. Berchtold, on verra l'explication qu'il propose du détour de Fortinbras, cet autre vengeur, par la Pologne, qui n'est pas le chemin le plus court pour se rendre de Norvège en Danemark. Mais Polonius/Polonia : même champ sémantique.

On aura noté au passage que sainte Gertrude de Nivelle était aussi révérée en Brabant que dans les autres provinces francophones des Pays-Bas, et l'on sait que nonobstant l'homonymie avec la famille maternelle de Shakespeare, la Forêt d'Ardennes où les protagonistes de As You Like It se retrouvent est située non loin du Brabant et de la Wallonie. Dans Love's Labours Lost le compagnon du roi de Navarre, Biron, se souvient d'avoir dansé avec Rosaline dans le Brabant, et un duc de Brabant est cité à plusieurs reprises dans Henry $V$, mais il n'est pas exclu que, dans Othello Shakespeare ait recherché un rapprochement de sonorités entre Brabantio et brabble afin de signifier la faiblesse, voire la sénilité du vieillard, marquée notamment dans son élocution. Telle est bien la manière de dérivation observée dans The Tempest, pour le nom d'un personnage comme Trinculo; plutôt que d'argot napolitain, ne s'agirait-il pas ici d'un dérivé de l'allemand trinken, si proche de to drink qu'il est immédiatement intelligible à l'oreille anglaise, en particulier au cours de la scène ii de l'acte II, où Stephano réconforte son compagnon d'infortune de la manière que l'on sait. D'une simple remarque, l'auteur le rapproche de son public en lui faisant prononcer :

Were I in England now as once I was! Tempest, II, ii, 1.28.

Toutefois, à la différence du "drunken butler", Trinculo est donné comme "a jester", et il va nous permettre, avant de conclure, 
de voir comment sont nommés les "fous", soit les bouffons et autres personnages destinés à divertir les princes.

Dans A Midsummer Night's Dream Puck a deux noms, et le second précise bien son emploi : Robin Goodfellow; à lui de faire le Roger Bontemps, d'être le boute-en-train de la cour où Titania n'est pas absolument l'incarnation de la gaîté. Il faut reconnaître que les fantaisies et plaisanteries de son mari ne sont pas toujours du meilleur goût. Feste, le "clown" de Twelfth Night n'est nommé qu'une fois, mais ce nom dit assez ce qu'on attend de lui, l'atmosphère festive qui se doit de lutter contre les méfaits du puritanisme ambiant. Cependant les indications scéniques, et Olivia (Take away the fool, gentlemen!), le désignent sans autre forme de procès comme "fool", ce qui permet à Malvolio de déclarer sentencieusement :

Infirmity, that decays the wise, doth ever make the fool better. et de s'attirer la réplique :

God send you, sir, a speedy infirmity, for better increasing your folly! I, v, 74-76.

Le nom de Touchstone dans As You Like It implique bien l'élément essentiel révélateur du diseur de vérités commun à tous les bouffons de Cour, royale ou seigneuriale; et bien que la savante éditrice de la pièce dans la Collection The Arden Shakespeare constate :

"The early descriptions of Touchstone as "the clovnish fool" or "the roynish fool" introduce him as a rather uncouth character, and not at all as a sophisticated jester as Feste is.» (ed.cit., p. liv)

il est tout-à-fait dans son élément. Le bouffon de King Lear n'a pas de nom; il remplit sa fonction et disparaît bien avant la fin de la tragédie, s'étant entendu confier, suprême dérision :
$O$ fool, I shall go mad.
II, iv, 286.

et l'on sait de qui parle le vieux roi lorsqu'il doit constater:

and my poor fool is hang'd. $\quad \mathrm{V}$, iii, 306.

Le rôle de Lear's FOOL était destiné à l'acteur Robert Armin, pour qui Shakespeare avait aussi écrit celui de $F e s t e$, ce qui tendrait à prouver, indépendamment de la plasticité inhêrente à tout bon acteur comique, que le dramaturge ici voit essentiellement la fonction, le rôle dans la tragédie, et l'apparente désinvolture avec 
laquelle il se débarrasse du personnage à mi-parcours est assez significative.

Ces quelques propos sur les noms que Shakespeare utilise dans ses pièces nous semblent de nature à souligner l'un des aspects bien connus de la création chez les acteurs/auteurs.

Molière accusé de plagiat aurait déclaré : «Je prends mon bien où je le trouve». On en peut dire autant de Shakespeare : il tisse chemin faisant des réseaux d'allusions en manipulant des mots, que ceux-ci lui soient imposés par la fable qu'il a choisi de porter sur la scène, ou qu'il les fabrique pour les besoins de la cause, soit encore qu'il les emprunte, - et alors sa désinvolture n'est la plupart du temps qu'apparente. En cette attention aux mots, qu'il laisse briller de tous leurs feux, comme on voit palpiter les cristaux d'un lustre, qu'il joue de leurs irisations, qu'il nous en fasse saisir les harmoniques les plus ténues, c'est le poète qui s'affirme : depuis quatre cents ans spectateurs et lecteurs en demeurent séduits et éblouis.

Jean DUBU

Professeur honoraire de Première Supérieure

NOTES

1.1 HIV, III.iii.52.

2. Montaigne, op.cit., Paris 1992, p.64.

3. Encyclopedia Britannica, art. Scaliger Josephus Justus.

4. Jean Dubu, Du texte à la scène, Société française Shakespeare, Paris 1983, p.35-47.

5. Jacques Berchtold, Des Rats et des Ratières, Genève, 1992, p.106 et sqq.

6. Ibid., p.128-129.

7. Ibid., p.136.

8. Ibid., p 143. 